\title{
Aetiology of Tick-Borne Infections in an Adult Swedish Population-Are Co-Infections with Multiple Agents Common?
}

\author{
Marika Nordberg1 ${ }^{*}$, Pia Forsberg1,2, Johan Berglund ${ }^{3}$, Anneli Bjöersdorff4, Jan Ernerudh5, \\ Ulf Garpmo6, Mats Haglund7, Kenneth Nilsson ${ }^{8}$, Ingvar Eliasson ${ }^{9}$ \\ ${ }^{1}$ Infectious Medicine, Department of Clinical and Experimental Medicine, Faculty of Health Sciences, \\ Linköping University, Linköping, Sweden \\ ${ }^{2}$ Department of Infectious Diseases, County Council of Östergötland, Linköping, Sweden \\ ${ }^{3}$ Blekinge Institute of Technology, School of Health Science, Karlskrona, Sweden \\ ${ }^{4}$ Djursjukhusgruppen, Danderyd, Sweden \\ ${ }^{5}$ Clinical Immunology, Department of Clinical and Experimental Medicine, Faculty of Health Sciences, Linköping \\ University, Department of Clinical Immunology and Transfusion Medicine, County Council of Östergötland, \\ Linköping, Sweden \\ ${ }^{6}$ Department of Clinical Microbiology, Kalmar County Hospital, Kalmar, Sweden \\ ${ }^{7}$ Department of Infectious Diseases, Kalmar County Hospital, Kalmar, Sweden \\ ${ }^{8}$ Unit of Clinical Microbiology/Infectious Diseases, Department of Medical Sciences, Uppsala University, \\ Uppsala, Sweden \\ ${ }^{9}$ Department of Laboratory Medicine, Norra Älvsborg County Hospital, Trollhättan, Sweden \\ Email: ${ }^{*}$ marika.karlsson@liu.se
}

Received 17 January 2014; revised 17 February 2014; accepted 24 February 2014

Copyright (C) 2014 by authors and Scientific Research Publishing Inc.

This work is licensed under the Creative Commons Attribution International License (CC BY). http://creativecommons.org/licenses/by/4.0/

(c) (i)

\section{Abstract}

In Scandinavia, tick-borne infections affecting humans include Lyme borreliosis (LB), tick-borne encephalitis (TBE) and human granulocytic anaplasmosis (HGA). Each of these infections can present with unspecific symptoms. In this prospective clinical study, we recruited patients based on two independent inclusion criteria; 1) patients with unspecific symptoms, i.e. fever $\left(\geq 38.0^{\circ} \mathrm{C}\right)$ or a history of feverishness and/or any combination of headache, myalgia or arthralgia and 2) patients with erythema migrans (EM), following an observed tick bite or tick exposure within one month prior to onset of symptoms. A total of 206 patients fulfilled the study. Among these, we could identify 186 cases of LB (174 with EM), 18 confirmed and two probable cases of HGA and two cases of TBE. Thirteen of the HGA cases presented without fever. Furthermore, 22 of the EM patients had a

"Corresponding author.

How to cite this paper: Nordberg, M., Forsberg, P., Berglund, J., Bjöersdorff, A., Ernerudh, J., Garpmo, U., Haglund, M., Nilsson, K. and Eliasson, I. (2014) Aetiology of Tick-Borne Infections in an Adult Swedish Population-Are Co-Infections with Multiple Agents Common? Open Journal of Clinical Diagnostics, 4, 31-40. http://dx.doi.org/10.4236/ojcd.2014.41007 
sub-clinical co-infection with Anaplasma phagocytophilum, based on serology. Both TBE cases had co-infections, one with Borrelia burgdorferi and one with Anaplasma phagocytophilum. We conclude that it is important to consider several causative agents and possible co-infections in the clinical management of infectious diseases where ticks may be suspected as vectors.

\section{Keywords}

\section{Epidemiology; Tick-Borne Infections; Tick-Borne Encephalitis; Lyme Borreliosis; Human Granulocytic Anaplasmosis}

\section{Introduction}

Hard ticks, in Sweden mainly Ixodes ricinus, transmit several zoonotic agents, the most well-known being bacteria Borrelia burgdorferi sensu latu (B. burgdorferi), Anaplasma phagocytophilum (A. phagocytophilum) and tick-borne encephalitis (TBE) virus. Each of these causative agents can initially induce fever and unspecific symptoms, including headache, myalgia and arthralgia [1]-[6].

Ixodes ricinus is distributed mainly in the south and central parts of Sweden, and along the east-coast up to Umeå. This coincides with the distribution of borreliosis and anaplasmosis, while TBE cases are distributed in a patchier, geographically localised way [7]. Recently, new endemic areas of TBE virus have been identified in southern Sweden [8].

This prospective clinical multi-centre study in the southeast part of Sweden was designed to estimate the extent to which tick-associated unspecific symptoms or fever could be attributed to B. burgdorferi, A. phagocytophilum, or TBE virus. In addition, we wanted to reveal co-infections with these agents in patients with localised Borrelia infection, i.e. erythema migrans (EM).

\section{Materials and Methods}

\subsection{Patients}

During the tick-active season of May $1^{\text {st }}$ through December $31^{\text {st }}$, 2001, 250 patients $\geq 15$ years old were recruited from 31 primary health care centres and departments of infectious diseases in the counties of Blekinge, Kalmar and Östergötland in southeast Sweden. The patients were categorized into four age groups, 15 - 20, 21 40, $41-60$ and $\geq 61$ years of age. Inclusion criteria were as follows: a) Unspecific symptoms, i.e. fever $\left(\geq 38.0^{\circ} \mathrm{C}\right)$ or a history of feverishness and/or any combination of headache, myalgia or arthralgia, or b) erythema migrans (EM), following an observed tick bite or tick exposure within one month prior to onset of symptoms. Patients with obvious signs of other concurrent infections, e.g. respiratory tract infection or urinary tract infection, or other obvious diseases that could complicate clinical assessment were not included.

\subsection{Healthy Controls}

Sera from 150 healthy blood donors, 50 from each County, were investigated for the presence of antibodies against B. burgdorferi (IgM + IgG) and A. phagocytophilum (IgG only). The control group was not investigated with TBE virus serology.

\subsection{Collection of Clinical and Laboratory Data}

At enrolment, the including physician used a standardized questionnaire to record patient history, as well as clinical and epidemiological findings. Two to three weeks after the initial visit, the patients were interviewed over the phone by a clinical research nurse, using a standardized follow-up questionnaire.

At enrolment, blood was drawn for serological investigations (B. burgdorferi, A. phagocytophilum, TBE virus) and polymerase chain reaction (PCR) (A. phagocytophilum). Blood samples for serological follow-up were collected at 6 - 8 weeks and six months after enrolment. For patients with positive Anaplasma-serology an additional serology was performed 12 months after enrolment.

Further blood samples were collected according to a standardized protocol at enrolment and after 6 - 8 weeks 
for analysis of complete blood count, serum aspartate aminotransferase, alanine aminotransferase, lactate dehydrogenase and C-reactive protein.

\subsection{Case Definitions}

\subsubsection{B. burgdorferi}

Laboratory evidence of clinical or subclinical infection with B. burgdorferi was defined as follows, based on comparison of acute and convalescent sera, using Borrelia-specific antibody tests:

a) seroconversion (IgM or IgG) in the ELISA

or

b) significant rise in IgG concentration in the ELISA

or

c) appearance of new, significant bands in the Western Blot (WB) banding pattern between acute and early or late convalescent sera.

Clinical infection, i.e. clinical disease caused by B. burgorferi was defined as i) laboratory evidence of infection in combination with one or several of the following symptoms, headache, myalgia, arthralgia, fever, or ii) EM with or without these symptoms or laboratory evidence.

\subsubsection{A. phagocytophilum}

Laboratory evidence of clinical or subclinical infection with A. phagocytophilum was defined as follows, based on a comparison of acute and convalescent sera or a gene amplification test (PCR) of blood collected at the initial visit:

Confirmed:

a) IgG antibody seroconversion i.e. from negative to positive titre

or

b) at least a four-fold rise in IgG antibody titre

or

c) positive A. phagocytophilum PCR

Probable:

a) a permanently high IgG antibody titre of $\geq 640$

or

b) at least a four-fold decrease in IgG antibody titre during the investigation period of 12 months.

Human granulocytic anaplasmosis (HGA) i.e. clinical disease caused by A. phagocytophilum, was defined as laboratory evidence of infection in combination with concurrent clinical disease, i.e. fever, myalgia, arthralgia and/or headache. All other infections with A. phagocytophilum were considered as subclinical [3] [9].

\subsubsection{TBE Virus}

Infection with TBE virus was defined as a positive IgM screen which was confirmed in the specific Rapid Fluorescent Focus Inhibition Test (RFFIT) [10]. Infection in combination with relevant clinical symptoms was considered as clinical TBE.

\subsection{Serology}

\subsubsection{B. burgdorferi}

Sera were tested for B. burgdorferi IgG and IgM antibodies by a commercial ELISA (Genzyme Virotech GmbH, Germany). Sera were absorbed with RF sorbotech (Genzyme Virotech) before testing for IgM antibodies. Positive or equivocal samples from ELISA were further tested by WB (Genzyme Virotech GmbH, Germany), using an AutoBlot 36 (GeneLabs Diagnostics, USA). Evaluation was performed according to the instructions of the manufacturer.

\subsubsection{A. phagocytophilum}

IgG antibody titres to A. phagocytophilum were determined by an indirect immunofluorescense assay (IFA) using A. phagocytophilum IgG test kit (Focus technologies, Cypress, California, USA) according to the instructions of the manufacturer. A titre of $\geq 1: 80$ was considered as positive. 


\subsubsection{TBE-Virus}

Sera from the acute phase were tested for anti-TBE virus antibodies (IgM and IgG) with an ELISA (Immunozym FSME IgM and IgG, respectively, Progen Biotechnik GmbH, Germany). The evaluation was made according to the manufacturer's instructions. Both IgM- and IgG-positive samples were submitted to the Swedish Institute for Infectious Disease Control for confirmation by a rapid fluorescent focus inhibition test (RFFIT) [10].

\subsection{DNA-Extraction and Gene Amplification}

DNA-extraction (No 1) A. phagocytophilum

DNA was extracted from EDTA blood with the QIAamp Tissue Kit using the protocol recommended by the manufacturer (Qiagen Inc, USA).

DNA extraction (No2) A. phagocytophilum

Two hundred microlitres of human EDTA blood was extracted and tested for the presence of A. phagocytophilum DNA in a KingFisher mL automated extractor (Thermo Electron Corporation, USA) by BioSprint DNA Blood Kit (Qiagen Inc, USA) which is designed for purification of total DNA.

A negative and a positive control sample were included in every run. All samples were stored at $-20^{\circ} \mathrm{C}$ until analyzed by PCR.

PCR amplification (No1) A. phagocytophilum

A nested PCR assay targeting the 16S rRNA gene was performed as previously described [11]. EDTA blood from a clinically ill and A. phagocytophilum inclusion-positive cow was used as a positive control, and EDTA blood from a healthy human blood donor was used as a negative control. PCR amplifications were performed in a Perkin Elmer 9600 thermal cycler, using reagents from the Gene Amp PCR kit Amplitaq DNA Polymerase (Perkin Elmer, Applied Biosystems Division, Foster City, CA, USA).

PCR amplification (No2) A. phagocytophilum

PCR for detection of A. phagocytophilum was conducted by using MSP2-3F (5'-CCAGCGTTTAGCAAGA TAAGAG) and MSP2-3R (5'-GCCCAGTAACAACATCATAAGC) directed towards the major surface protein 2 of A. phagocytophilum [12].

The products were visualized by electrophoresis in a $2 \%$ agarose gel stained with ethidium bromide. Negative and positive controls were included in all PCR runs.

\subsection{Treatment}

Medical treatment was decided by the physician according to international recommendations and was not a part of the study protocol [13] [14].

\subsection{Ethics}

The Research Ethics Committees of the Universities of Lund and Linköping approved the study (Nr 01-133).

\section{Results}

A total of 250 individuals volunteered to participate in this study, out of which 206 (110 women and 96 men) completed the study (Table 1$)$. Forty-four patients were excluded due to drop-out $(n=42)$ or because they received another diagnosis $(n=2)$. The majority $(80 \%)$ were above 40 years of age (data not shown). The geographic distribution of cases was County of Blekinge $(n=102)$, Kalmar $(n=38)$ and Östergötland $(n=66)$, all situated in southeast Sweden.

Figure 1, Tables 1 and 2 show different aspects of the relations between symptoms, EM and Borrelia and Anaplasma serology. In the total material of 206 patients, 32 were recruited on the bases of unspecific symptoms in conjunction with a tick bite, while 174 patients were recruited on the basis of EM. In the EM group, 65 patients simultaneously suffered from unspecific symptoms with or without fever (Table 1).

We found a total of 42 cases with serological evidence of a concurrent infection with A. phagocytophilum (Table 1, Figure 1). Twenty of these had clinical HGA (Figure 1). Eighteen of these were confirmed and two probable, according to our definitions (Table 2). Thus, the rate of HGA cases in the total material was $10 \%$. Among the 20 HGA cases (Figure 1, Table 2), 13 patients presented with headache or myalgia and/or arthralgia, but without any fever, according to patient history or determined at presentation. The remaining seven patients 
Table 1. Symptoms of the 206 included study patients, and their relation to Anaplasma and Borrelia infection. One hundred and nine of the 174 patients with clinical signs of EM had no other recorded clinical symptoms at the time of inclusion. Of these 109 patients, 22 had serological evidence of current A. phagocytophilum infection, i.e. subclinical Anaplasma infection. In the group with EM and unspecific symptoms, 14 patients had concurrent serological evidence of A. phagocytophilum infection, i.e. by definition human granulocytic anaplasmosis (HGA). Six further cases of HGA were identified in the symptomatic group without EM. Out of 174 patients with EM, 74 (43\%) had serological evidence of current Borrelia infection. Twelve of the patients with unspecific symptoms but without EM also had serological evidence of current Borrelia infection. Of these, two patients had concurrent HGA.

\begin{tabular}{|c|c|c|c|c|c|}
\hline \multirow[t]{2}{*}{ Signs/symptoms } & \multicolumn{3}{|c|}{ Serological status } & \multicolumn{2}{|r|}{ Total, $\mathrm{n}$} \\
\hline & $\begin{array}{c}\text { Anaplasma- } \\
\text { Borrelia- } \\
n=96\end{array}$ & $\begin{array}{c}\text { Anaplasma- } \\
\text { Borrelia }^{+} \\
\mathrm{n}=68\end{array}$ & $\begin{array}{c}\text { Anaplasma+ } \\
\text { Borrelia- } \\
n=24\end{array}$ & $\begin{array}{c}\text { Anaplasma+ } \\
\text { Borrelia+ } \\
n=18\end{array}$ & 206 \\
\hline Only EM & 59 & 28 & 11 & 11 & 109 \\
\hline EM + unspecific symptoms & 21 & 30 & 9 & 5 & 65 \\
\hline Unspecific symptoms (no EM) & 16 & $10^{*}$ & $4^{*}$ & 2 & 32 \\
\hline
\end{tabular}

$\mathrm{n}$ = number. Anaplasma = Anaplasma phagocytophilum. Anaplasma $-=$ No serological evidence of antibodies. Anaplasma $+=$ Serological evidence of antibodies. Borrelia = Borrelia burgdorferi. Borrelia $-=$ No serological evidence of antibodies. Borrelia $+=$ Serological evidence of antibodies. $\mathrm{EM}=$ Erythema migrans. Unspecific symptoms = Fever and/or myalgia and/or arthralgia and/or headache. ${ }^{*}=$ one concurrent TBE case included.

Table 2. Symptoms, signs and serological findings of the 20 HGA cases, number 1 - 18 confirmed and number 19 - 20 probable.

\begin{tabular}{|c|c|c|c|c|c|c|c|c|c|c|c|c|}
\hline No. & Sex & Age & Tick & Headache & Myalgia/ & Fever & EM & $B b$ serology & A. phagocy & ytophilum se & erology & \\
\hline & & group & bite & & arthralgia & & & & At enrolment & 2 - 3 weeks & 6 months & 1 year \\
\hline 1 & $\mathrm{~F}$ & 2 & Yes & + & + & + & - & - & Neg & 80 & Neg & ND \\
\hline 2 & F & 3 & Yes & + & + & + & - & - & Neg & 80 & ND & Neg \\
\hline 3 & M & 3 & $>1$ bite & + & - & - & - & - & Neg & 80 & 80 & Neg \\
\hline 4 & M & 2 & $>1$ bite & + & - & - & - & - & Neg & 80 & 80 & 160 \\
\hline 5 & M & 4 & Yes & - & + & - & - & + & Neg & 160 & ND & 320 \\
\hline 6 & M & 3 & Yes & + & - & + & - & + & Neg & 80 & Neg & ND \\
\hline 7 & F & 4 & Yes & + & - & - & + & - & Neg & Neg & 160 & ND \\
\hline 8 & $\mathrm{~F}$ & 4 & Yes & + & - & - & + & - & 160 & 160 & 640 & 1280 \\
\hline 9 & $\mathrm{~F}$ & 4 & Yes & + & - & - & + & - & Neg & Neg & 80 & Neg \\
\hline 10 & F & 3 & Yes & + & - & - & + & - & Neg & Neg & 80 & 80 \\
\hline 11 & M & 1 & Yes & - & + & - & + & - & Neg & Neg & 80 & ND \\
\hline 12 & M & 3 & Yes & - & + & - & + & - & Neg & 80 & Neg & Neg \\
\hline 13 & M & 2 & Yes & + & - & + & + & - & Neg & 80 & Neg & ND \\
\hline 14 & $\mathrm{~F}$ & 3 & Unknown & + & - & - & + & + & Neg & 40 & 80 & 160 \\
\hline 15 & M & 3 & Yes & + & - & + & + & + & Neg & 160 & 80 & 80 \\
\hline 16 & M & 3 & Yes & - & + & + & + & + & Neg & 80 & Neg & ND \\
\hline 17 & F & 4 & Unknown & + & - & + & + & + & 40 & Neg & 80 & ND \\
\hline 18 & F & 3 & Unknown & + & - & - & + & + & 40 & Neg & 160 & 80 \\
\hline 19 & $\mathrm{~F}$ & 3 & Unknown & + & + & - & + & - & 640 & 320 & Neg & 160 \\
\hline 20 & M & 3 & Yes & + & - & - & + & - & 640 & 640 & 320 & 640 \\
\hline
\end{tabular}

$\mathrm{F}=$ Female, $\mathrm{M}=$ Male. Age group $1=15-20$ years. Age group $2=21-40$ years. Age group $3=41-60$ years. Age group $4=61$ or older. EM = erythema migrans, $\mathrm{Pos}=$ positive serology, Neg = negative serology, $\mathrm{ND}=$ not done, $\mathrm{Bb}=$ Borrelia burgdorferi, Ap = Anaplasma phagocytophilum, $+=$ positive, $-=$ negative. 


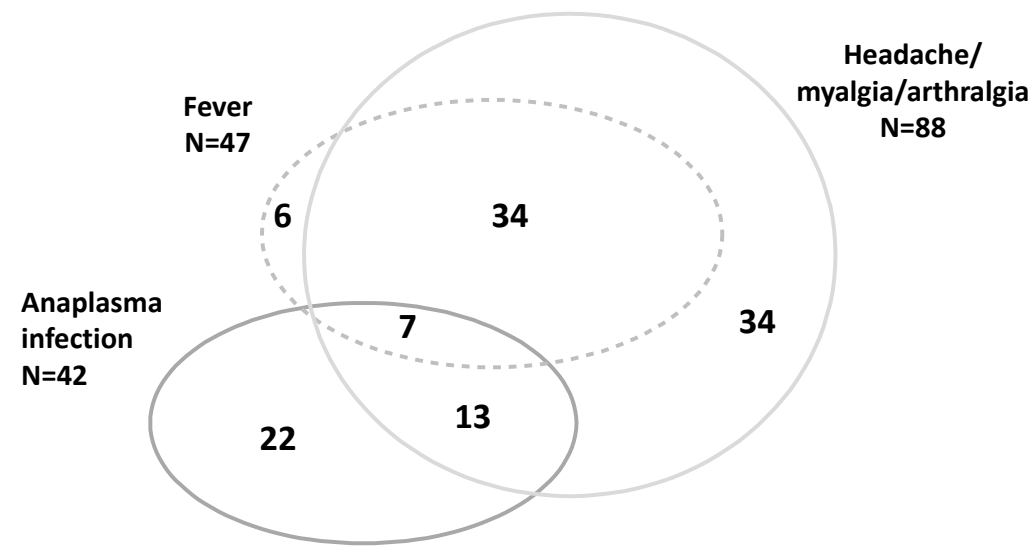

Figure 1. The relation between A. phagocytophilum infection ( $\mathrm{n}=42$, fat grey circle) and headache, myalgia, arthralgia ( $\mathrm{n}=88$, thin grey circle) or fever ( $\mathrm{n}=$ 47 , grey dots) suggestive of human granulocytic anaplasmosis (HGA). Twenty patients $(10 \%, n=7+13)$ could be identified as HGA cases, according to our definitions. Out of the 20 HGA cases, only seven presented with fever. In addition, 22 patients had serological evidence of a sub-clinical A. phagocytophilum infection (fat grey cricle).

presented with fever. No patients were PCR-positive for A. phagocytophilum. Twelve of the 18 confirmed cases of HGA and both the probable ones were found in the EM group (Table 2). All patients but four had one or more known tick bites. Three of the HGA patients (Table 2, patient no 13, 16, 17) had no symptoms at the time of inclusion but developed symptoms one to two days later according to the interview-based follow-up.

HGA cases were found in all age groups. None of the cases was severe and no HGA-positive patients required hospitalization. Only six patients with confirmed HGA had moderately elevated transaminase levels, while no patient had thrombocytopenia and only one had leukocytopenia. Four patients had elevated levels of C-reactive protein (data not shown). At the interview-based follow-up (median time 25 days from first visit), twelve of the HGA patients were completely recovered while three still had headache as a single symptom, and the other five still had headache, myalgia or neurological symptoms. Another 22 patients (included due to EM) showed serological evidence of current Anaplasma infection (Table 1). They did not develop any other clinical symptoms according to the follow-up interview, and thus were defined as having subclinical Anaplasma infection. Eleven of these 22 co-infected patients, i.e. those having EM plus Anaplasma antibodies, also had serological evidence of $B$. burgdorferi (Table 1 ).

Of the 206 study patients, 174 presented with EM. Of these, 36 patients (21\%) also had serologic evidence of A. phagocytophilum infection (Table 1). A total of 186 patients had a current infection with Borrelia, i.e., EM or serological evidence of Borrelia infection, or both (Table 1). Of the 174 patients with EM, $43 \%(n=74)$ also developed Borrelia-specific antibodies. The age distribution of EM, fever and headache/myalgia/arthralgia is shown in Figure 2.

In total, two patients (1\%) had conclusive serological evidence of current TBE infection, with positive IgM + IgG serology and positive RFFIT. One of these patients was co-infected with A. phagocytophilum, while the other had serological evidence of an infection with B. burgdorferi. Both presented with headache and myalgia/ arthralgia that had been experienced for one month. The patient with HGA had a biphasic course and also a history of fever that had lasted one month. A lumbar puncture showed elevated mononuclear cells $\left(150 \times 10^{6} / \mathrm{L}\right)$ in the cerebrospinal fluid. She developed neurological symptoms (cognitive problems, fatigue) that were considered moderate at a check-up two months after onset of symptoms. The other patient presented with fever that had lasted one week, and tremor and vertigo that had lasted four weeks. No lumbar puncture was done. The symptoms declined after about one month.

Furthermore, another 14 patients were IgG positive for TBE virus, out of which five were RFFIT-positive. Four of the RFFIT-positive and one of the RFFIT-negative patients had been immunized with TBE vaccine according to the questionnaire. Another five patients who reported previous immunization were IgG negative. Among the patients with positive IgG and negative RFFIT test, four had a history of immunization with yellow fever or Japanese B encephalitis. One non-immunized patient was IgG and RFFIT-positive but lacked clinical- 


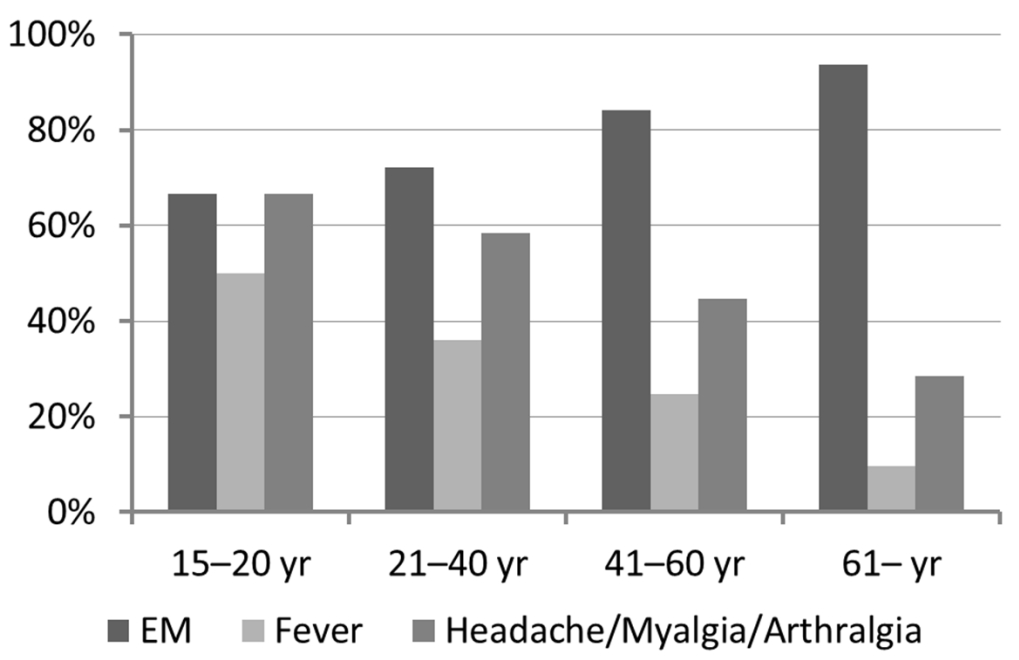

Figure 2. Clinical findings and age distribution of the relative rate of erythema migrans, fever and headache, myalgia and arthralgia in the total material of 206 patients. Age distribution, $15-20$ years $(n=6), 21-40$ years $(n=36), 41-60(n$ $=101), \geq 61$ years $(n=63)$.

symptoms. This may represent a previous or a current subclinical infection.

The seroprevalence in healthy blood donors was $13 \%$ for $A$. phagocytophilum (IgG) and $8 \%$ for $B$. burgdorferi serology (IgG or IgM or both) (data not shown). Blood donors were not screened with TBE virus serology.

\section{Discussion}

To our knowledge this is the largest prospective clinical investigation focusing on mixed aetiology in tick-borne infections in Europe. Some new and interesting results should be pointed out. First of all, this investigation revealed strong evidence for subclinical co-infections with A. phagocytophilum in patients with EM. Altogether, $20 \%$ of the 109 EM patients without other symptoms had serological evidence of sub-clinical co-infection with A. phagocytophilum (Table 1). Taking all the $174 \mathrm{EM}$ patients into account, including the 65 with symptoms of disseminated infection (unspecific symptoms with or without fever), the rate of co-infection was still similar (21\%). It has been argued that there may be cross-reactivity between Borrelia and Anaplasma serology [15], which could explain this phenomenon, while Bunnell et al. found no serological cross-reactions in infection of laboratory mice [16]. Co-infections may actually be the most probable explanation. We did not find any PCRpositive cases of HGA to support this, but the low rate of PCR positivity in HGA has been a general problem in our previous investigations, as well as in other investigations in Europe [13] [17]-[19]. We believe that this is not a methodological problem, because we have good experience from veterinary specimens and always include proper controls. An alternative explanation may be that the bacterial count seems to be very low at the time when patients decide to consult their physician [20]. Bakken et al. suggested that the IFA of the paired acute phase and convalescent phase serum is more sensitive compared to PCR or culture of HGA [21]. Thus, a negative PCR result does not rule out HGA diagnosis, if other criteria are fulfilled, based on current guidelines from both Europe and the USA [3] [21].

Another observation concerns the clinical signs presented by the 20 patients (Tables 1 and 2) who had not only serological evidence of A. phagocytophilum infection but also clinical symptoms, consistent with HGA. They showed a different clinical spectrum from what is usually reported in the literature. Only one third of the HGA patients in our material had a history of fever (Figure 1, Table 2). As fever has been an inclusion criterion in most prospective HGA studies so far, patients without fever have usually not been discovered or reported. Typically, HGA has been described as an acute non-specific febrile illness with fever $>39^{\circ} \mathrm{C}$, myalgia and malaise [22]. In a meta-analysis of clinical manifestations in patients with HGA from North America and Europe, fever was found in 92\% of cases [23]. Other European studies report fever in $100 \%$ of the cases [13] [17] [18] [24] [25], which may indicate that fever has been an inclusion criteria.

Our study indicates that a patient with both EM and clinical symptoms can easily be interpreted as a clean-cut 
case of Lyme borreliosis, while it may actually constitute a true clinical case of HGA, mixed with EM due to a co-infection with Borrelia. On the other hand, a sub-clinical co-infection with A. phagocytophilum can be interpreted as clinical HGA, due to symptoms actually caused by concurrent Borrelia infection.

Only two of the seven patients with HGA and fever did not have any signs of Borrelia infection (Table 2). Four of the seven patients had EM and four of them had serological signs of current Borrelia infection. Interestingly, only 7/20 patients in the HGA group had serological evidence of concurrent Borrelia infection (Table 2). As it is expected that patients with disseminated Borrelia infection should react serologically, seronegativity towards Borrelia (even after six months) may support the fact that the clinical symptoms were caused by the Anaplasma infection.

Taken together, it is clearly not a straightforward task, or even possible at all, to ascertain the causative etiologic agent, i.e. Anaplasma and/or Borrelia, and thus to select the appropriate treatment. Co-infections among patients with EM are probably quite common, but the reported frequencies vary [17] [26]. Although reported cases of HGA often have deranged laboratory parameters, laboratory investigations added little value in our study [17] [22]. It also seems that the HGA cases often are self-limiting and milder in Europe [27].

A more pragmatic approach is therefore needed. If treatment of a tick-associated infection with symptoms like fever and/or myalgia, arthralgia or headache is indeed considered, doxycyclin should be the drug of choice, as it is effective against both B. burgdorferi and A. phagocytophilum. [3] [13] [20] [28].

TBE is a growing health problem, with about 3000 cases annually in Europe and 174 cases reported in Sweden in 2010 [29] [30]. Two patients with clinical TBE were found in this study, both infected in known endemic areas within the county of Östergötland. Both cases had co-infections, one with Anaplasma and one with Borrelia, and were discovered in the early clinical phase with unspecific symptoms. It is important to diagnose these patients because the disease might be severe with up to $40 \%$ sequelae [31].

LB is the most common tick-transmitted disease in the northern hemisphere [5] and in Sweden [32]. In this study a total of 186 patients had Borrelia infection, i.e. EM or serological evidence of a current Borrelia infection. In accordance with other studies, [32], only $43 \%$ of the 174 patients with EM had serological evidence of $B$. burgdorferi infection. It has been reported that unspecific symptoms are common in LB and that they can be presented without a typical EM [33]. However, most of our patients were recruited by the inclusion criteria EM with or without other symptoms, making it difficult to draw any general conclusions concerning the prevalence of unspecific symptoms in LB patients.

Nevertheless, it should be noted that there was an obvious age bias in the distribution of fever and unspecific symptoms (Figure 2).

\section{Conclusion}

The majority of the patients included had Lyme borreliosis (90\%). This was probably a result of the fact that EM was one of the inclusion criteria and the areas studied are known to be endemic for this infection. The majority (13/20) of the cases with HGA presented with myalgia, arthralgia and/or headache but no reported fever. Interestingly, only seven patients had a significant fever reaction, which is notable since fever has been an inclusion criterion in many reports [17]. The study shows that unspecific symptoms following a tick bite in the southeast of Sweden may be caused by B. burgdorferi, A. phagocytophilum or TBE virus, or a mixed infection with several of these agents. One should keep this in mind when considering diagnostic procedures and therapy regarding tick-borne infections.

\section{Acknowledgements}

Parts of the results were presented at the $10^{\text {th }}$ International Conference on Lyme Borreliosis and Other Tickborne Diseases, Vienna, Austria, 2005. We would like to thank all physicians and nurses from the participating primary care centres and Departments of Infectious diseases in Blekinge, Kalmar and Östergötland. We also would like to express our gratitude to the clinical research nurses, Lotta Lindvall, Linköping, Karin Holmkvist, Blekinge and Kerstin Glebe, Kalmar, Louise Stjernberg, Ph.D., Blekinge for her help, Lic. Med. Sci. Sirkka Vene, Swedish Institute for Infectious Disease Control for running the TBE RFFIT-assays, and professor Christina Ekerfelt, Dept of Clinical and Experimental Medicine, Linköping University hospital, and Barbro H. Skogman, Ph.D., Center for Clinical Research Dalarna, Falun, Sweden, for critical advice. 


\section{Transparency Declaration}

This study was supported by grants from FORSS-The Health Research Council in the Southeast of Sweden (Grant No 2000-320, 2001-332, 2002-335) and research funds from the Östergötland County Council (Grant No 2000-027, 2001-015, 2002-031) and ALF grants from the Östergötland County Council. The authors declare that they have no conflicts of interest in relation to this work.

\section{References}

[1] Wahlberg, P., Salminen, A., Weckstroem, P. and Oker-Blom, N. (1964) Diphasic Tick-Borne Meningo-Encephalitis, Kumlinge Disease, in the Aland Islands. Diagnosis, Clinical Features, and Epidemiology. Acta Med Scand, 175, 275-286. http://dx.doi.org/10.1111/j.0954-6820.1964.tb04661.x

[2] Lotric-Furlan, S., Petrovec, M., Avsic-Zupanc, T. and Strle, F. (2000) Clinical Distinction between Human Granulocytic Ehrlichiosis and the Initial Phase of Tick-Borne Encephalitis. The Journal of Infection, 40, 55-58. http://dx.doi.org/10.1053/jinf.1999.0587

[3] Brouqui, P., Bacellar, F., Baranton, G., Birtles, R.J., Bjoersdorff, A., Blanco, J.R., et al. (2004) Guidelines for the Diagnosis of Tick-Borne Bacterial Diseases in Europe. Clinical Microbiology and Infection, 10, 1108-1132. http://dx.doi.org/10.1111/j.1469-0691.2004.01019.x

[4] Chen, S.M., Dumler, J.S., Bakken, J.S. and Walker, D.H. (1994) Identification of a Granulocytotropic Ehrlichia Species as the Etiologic Agent of Human Disease. Journal of Clinical Microbiology, 32, 589-595.

[5] Stanek, G. and Strle, F. (2003) Lyme Borreliosis. Lancet, 362, 1639-1647. http://dx.doi.org/10.1016/S0140-6736(03)14798-8

[6] Walker, D.H. and Dumler, J.S. (1996) Emergence of the Ehrlichioses as Human Health Problems. Emerging Infectious Diseases, 2, 18-29. http://dx.doi.org/10.3201/eid0201.960102

[7] Holmgren, E.B. and Forsgren, M. (1990) Epidemiology of Tick-Borne Encephalitis in Sweden 1956-1989: A Study of 1116 Cases. Scandinavian Journal of Infectious Diseases, 22, 287-295. http://dx.doi.org/10.3109/00365549009027050

[8] Fält, J., Lundgren, Å., Alsterlund, R., Carlsson, B., Eliasson, I., Haglund, M., et al. (2006) Tick-Borne Encephalitis (TBE) in Skane, Southern Sweden: A New TBE Endemic Region? Scandinavian Journal of Infectious Diseases, 38, 800-8004. http://dx.doi.org/10.1080/00365540600664068

[9] Bakken, J.S. and Dumler, S. (2008) Human Granulocytic Anaplasmosis. Infectious Disease Clinics of North America, 22, 433-448. http://dx.doi.org/10.1016/j.idc.2008.03.011

[10] Vene, S., Haglund, M., Vapalahti, O. and Lundkvist, A. (1998) A Rapid Fluorescent Focus Inhibition Test for Detection of Neutralizing Antibodies to Tick-Borne Encephalitis Virus. Journal of Virological Methods, 73, 71-75. http://dx.doi.org/10.1016/S0166-0934(98)00041-X

[11] Massung, R.F., Slater, K., Owens, J.H., Nicholson, W.L., Mather, T.N., Solberg, V.B., et al. (1998) Nested PCR Assay for Detection of Granulocytic Ehrlichiae. Journal of Clinical Microbiology, 36, 1090-1095.

[12] Massung, R.F. and Slater, K.G. (2003) Comparison of PCR Assays for Detection of the Agent of Human Granulocytic Ehrlichiosis, Anaplasma phagocytophilum. Journal of Clinical Microbiology, 41, 717-722. http://dx.doi.org/10.1128/JCM.41.2.717-722.2003

[13] Bjoersdorff, A., Berglund, J., Kristiansen, B.E., Soderstrom, C. and Eliasson, I. (1999) [Varying Clinical Picture and Course of Human Granulocytic Ehrlichiosis. Twelve Scandinavian Cases of the New Tick-Borne Zoonosis Are Presented]. Lakartidningen, 96, 4200-4204.

[14] Dumler, J.S. and Walker, D.H. (2001) Tick-Borne Ehrlichioses. The Lancet Infectious Diseases, 1, 21-28. http://dx.doi.org/10.1016/S1473-3099(09)70296-8

[15] Blanco, J.R. and Oteo, J.A. (2002) Human Granulocytic Ehrlichiosis in Europe. Clinical Microbiology and Infection, 8, 763-772. http://dx.doi.org/10.1046/j.1469-0691.2002.00557.x

[16] Bunnell, J.E., Magnarelli, L.A. and Dumler, J.S. (1999) Infection of Laboratory Mice with the Human Granulocytic Ehrlichiosis Agent Does Not Induce Antibodies to Diagnostically Significant Borrelia burgdorferi Antigens. Journal of Clinical Microbiology, 37, 2077-2079.

[17] Bjoersdorff, A., Wittesjo, B., Berglund, J., Massung, R.F. and Eliasson, I. (2002) Human Granulocytic Ehrlichiosis as a Common Cause of Tick-Associated Fever in Southeast Sweden: Report from a Prospective Clinical Study. Scandinavian Journal of Infectious Diseases, 34, 187-191. http://dx.doi.org/10.1080/00365540110080061

[18] Grzeszczuk, A., Ziarko, S., Kovalchuk, O. and Stanczak, J. (2006) Etiology of Tick-Borne Febrile Illnesses in Adult Residents of North-Eastern Poland: Report from a Prospective Clinical Study. International Journal of Medical Microbiology, 296, 242-249. http://dx.doi.org/10.1016/j.ijmm.2006.01.007 
[19] Lotric-Furlan, S., Petrovec, M., Avsic-Zupanc, T. and Strle, F. (2004) Comparison of Patients Fulfilling Criteria for Confirmed and Probable Human Granulocytic Ehrlichiosis. Scandinavian Journal of Infectious Diseases, 36, 817-822. http://dx.doi.org/10.1080/00365540410021171

[20] Bakken, J.S. and Dumler, J.S. (2006) Clinical Diagnosis and Treatment of Human Granulocytotropic Anaplasmosis. Annals of the New York Academy of Sciences, 1078, 236-247. http://dx.doi.org/10.1196/annals.1374.042

[21] Bakken, J.S., Haller, I., Riddell, D., Walls, J.J. and Dumler, J.S. (2002) The Serological Response of Patients Infected with the Agent of Human Granulocytic Ehrlichiosis. Clinical Infectious Diseases, 34, 22-27. http://dx.doi.org/10.1086/323811

[22] Bakken, J.S. and Dumler, J.S. (2000) Human Granulocytic Ehrlichiosis. Clinical Infectious Diseases, 31, 554-560. http://dx.doi.org/10.1086/313948

[23] Dumler, J.S., Choi, K.S., Garcia-Garcia, J.C., Barat, N.S., Scorpio, D.G., Garyu, J.W., Grab, D.J. and Bakken, J.S. (2005) Human Granulocytic Anaplasmosis and Anaplasma phagocytophilum. Emerging Infectious Diseases, 11, 1828-1834. http://dx.doi.org/10.3201/eid1112.050898

[24] Petrovec, M., Lotric Furlan, S., Zupanc, T.A., Strle, F., Brouqui, P., Roux, V. and Dumler, J.S. (1997) Human Disease in Europe Caused by a Granulocytic Ehrlichia Species. Journal of Clinical Microbiology, 35, 1556-1559.

[25] Karlsson, U., Bjoersdorff, A., Massung, R.F. and Christensson, B. (2001) Human Granulocytic Ehrlichiosis-A Clinical Case in Scandinavia. Scandinavian Journal of Infectious Diseases, 33, 73-74. http://dx.doi.org/10.1080/003655401750064130

[26] Steere, A.C., McHugh, G., Suarez, C., Hoitt, J., Damle, N. and Sikand, V.K. (2003) Prospective Study of Coinfection in Patients with Erythema Migrans. Clinical Infectious Diseases, 36, 1078-1081. http://dx.doi.org/10.1086/368187

[27] Strle, F. (2004) Human Granulocytic Ehrlichiosis in Europe. International Journal of Medical Microbiology, 293, 2735.

[28] Wormser, G.P., Dattwyler, R.J., Shapiro, E.D., Halperin, J.J., Steere, A.C., Klempner, M.S., et al. (2006) The Clinical Assessment, Treatment, and Prevention of Lyme Disease, Human Granulocytic Anaplasmosis, and Babesiosis: Clinical Practice Guidelines by the Infectious Diseases Society of America. Clinical Infectious Diseases, 43, 1089-1134. http://dx.doi.org/10.1086/508667

[29] Lindquist, L. and Vapalahti, O. (2008) Tick-Borne Encephalitis. Lancet, 371, 1861-1871. http://dx.doi.org/10.1016/S0140-6736(08)60800-4

[30] Department of Epidemiology at the Swedish Institute for Communicable Diseases. (2011) Surveillance Database (SmiNet). Epi-aktuellt 14, 10.

[31] Gunther, G., Haglund, M., Lindquist, L., Forsgren, M. and Skoldenberg, B. (1997) Tick-Bone Encephalitis in Sweden in Relation to Aseptic Meningo-Encephalitis of Other Etiology: A Prospective Study of Clinical Course and Outcome. Journal of Neurology, 244, 230-238. http://dx.doi.org/10.1007/s004150050077

[32] Berglund, J., Eitrem, R., Ornstein, K., Lindberg, A., Ringer, A., Elmrud, H., Carlsson, M., Runehagen, A., Svanborg, C. and Norrby, R. (1995) An Epidemiologic Study of Lyme Disease in Southern Sweden. The New England Journal of Medicine, 333, 1319-1324. http://dx.doi.org/10.1056/NEJM199511163332004

[33] Feder Jr., H.M., Gerber, M.A., Krause, P.J., Ryan, R. and Shapiro, E.D. (1993) Early Lyme Disease: A Flu-Like Illness without Erythema Migrans. Pediatrics, 91, 456-459. 